\title{
Synthesis of superheavy elements and theory of atomic nucleus
}

\author{
B.N.Kalinkin , F.A.Gareev
}

\section{BLTPH JINR, Dubna, Russia}

\begin{abstract}
The connection of synthesis of long-lived superheavy nuclei and existence of the island of stability with the choice of realistic nuclear potential is discussed: the behaviour of the average field of nuclei is an important element of nuclear models.

It is also shown that it is just Dubna that possesses the priority both in the recent synthesis of a superheavy nucleus with charge $Z=114$ and in its theoretical prediction made 35 years ago. Possible sizes of the island of stability of superheavy nuclei are considered.
\end{abstract}




\section{Introduction}

The synthesis recently performed for a superheavy long-lived nucleus (SHN) with the charge $Z=114$ [1] that is in a beyond transuranium region of mass numbers was not a great surprise. The possibility of its existence was theoretically predicted as early as in 1966 [2], i.e. long before experimental synthesis. Nevertheless, this event was met with great interest and produces a lively discussion. Recently, there appear papers [3] and reviews [4, 5] devoted to that regions of studies.

Unfortunately, in our opinion, some of that admit considerable inaccuracies, errors, and even distortions of the known historical facts. Their serious drawback is also the descriptive aspect that dominates the studies, but there is completely absent the accent on the important problem of significance of the experimental synthesis of SHN for the theory of atomic nuclei. But this should be the main purpose of experiment.

In this paper, we briefly formulate our point of view on the problem as a whole and its significance for the construction of nuclear theory

\section{Essence of the theoretical aspect of the problem}

The problem is directly connected with the experimental fact: nuclei with $Z, N=$ $8,20,28,50,82$ (and for neutrons with $N=126$ ) are the most stable to various decay modes. Interpretation of this phenomenon became possible where there appeared the shell model [6, 7], according to which "magic" numbers are occupation numbers of one-particle levels in nuclei, upon which the spectrum acquires a considerable energy gap, and the binding energy becomes the highest. Consequently the theoretically predicted existence of superheavy nuclei beyond the limits of the periodic Table should at least be based on the calculation of one-particle proton and neutron spectra for detecting considerable energy gaps in them. Then, on the basis of the structure of levels of the found new shell, one should estimate the SHN stability to the most probable decay mode- fission, i.e. determine the barrier $V_{f}$ for it.

Of course. the problem can immediately be "closed " if it is assumed that the next to the $Z=82$ proton closed shell is the $Z=126$ shell. And for the long time, this was the opinion of most physicists.

It was no mere chance that parameters of the widely known Nilsson scheme [8] constructed on the oscillatory potential were more often fitted so as to reproduce the shell $Z=126$ in the region of large $A$, i.e. the prediction was in fact substituted by the assumption of the Nilsson scheme being valid for the description of a system of SHN levels. However, as early as in the midsixties when interest in the [problem revived, some theoreticians understood that the oscillatory potential (the Nilsson scheme [8]) is not valid for this purpose. We think, the only advantage of it is the simplicity of wave functions of one-particle states. From a physical point of view, the largest drawback of it leading to a number of consequences is that it tends to infinity near the nuclear surface. As a result, the wave functions of the one-particle spectrum exhibit a wrong behavior at the boundary and periphery of a nucleus - in the region that decisively contributes to the probabilities of radiative transitions (the transition operator $r^{\lambda} Y_{\lambda, \mu}(\theta, \phi), \lambda=1,2,3, \ldots$ ) as well as of elastic, inelastic scattering, and other reactions. Therefore, large discrepancies are inevitable (sometimes, by an order of magnitude) with calculations on "correct" wave functions.

A serious drawback of that scheme is the necessity of changing the parameters of the potential and spin-orbital interaction when passing to a higher shell. Therefore, it is not surprising that the calculations of the spectrum in the region of superheavy nuclei based on the distant extrapolation 
of the parameters of the potential and spin-orbital interaction led to different magic numbers for $\mathrm{Z}$ and N. For instance, Nilsson et al. [8] obtained $Z=126, N=164,184$. There arose other solutions, as well. It is evident that this scheme cannot be recognized convinced and reliable, especially, for predictions.

An acceptable solution to the problem should use the realistic potential $V(r)$ as the mean nuclear field. The term "realistic" means in the case that: 1)The potential $V(r)$ should be finite in magnitude (this follows, for instance, from finiteness of such nuclear characteristics as the binding energy of a nucleon and the limiting Fermi energy in the ground state of nuclei; 2)near $r=R_{0}$ ( $r=R_{0}$ is the radius of a nucleus), . should be smeared owing to quantum fluctuations of the nucleon density (especially, weakly coupled nucleons) in the surface layer of a nucleus; $3)$ it is preferable to use the spin-orbital interaction $V_{S O}(r)$ its most justified form proposed by Heisenberg in analogy with the electromagnetic field [9]

$$
V_{S O}=\kappa(\vec{l} \bullet \vec{S}) \frac{1}{r} \frac{d V_{N}}{d r}
$$

4) the electric charge $Z e$ should be distributed over the nucleus. In the first approximation for the proton-nucleus Coulomb interaction, one can assume

$$
\begin{gathered}
V_{c}(r)=\frac{(Z-1) e^{2}}{r}\left[\frac{3}{2} \frac{r}{R_{0}}-\frac{1}{2}\left(\frac{r}{R_{0}}\right)^{3}\right] ; r \geq R_{0} \\
V_{c}(r)=\frac{(Z-1) e^{2}}{r} ; r \leq R_{0} .
\end{gathered}
$$

The most convenient form of the potential . in the case of spherical nuclei (magic and nearmagic ones) was suggested by Saxon and Wood [10]:

$$
V(r)=-V_{0}\left(1+\exp \frac{r-R_{0}}{a}\right)^{-1}, \quad R_{0}=r_{0} A^{1 / 3} .
$$

Studies performed in the framework of the shell model and scattering theory [6] on nuclei have shown that expressions (1) and (3) should be improved by taken account of the dependence of parameters $V_{0}$ and $\kappa$ on $N$ and $Z$ as follows:

$$
V^{N, Z}(r)=-V_{0}^{N, Z}\left(1+\exp \frac{r-R_{0}}{a}\right)^{-1}, \quad R_{0}=r_{0} A^{1 / 3}
$$

where

$$
\begin{gathered}
V^{N}=V_{0}\left[1+0.63 \frac{N-Z}{A}\right], V^{Z}=V_{0}\left[1-0.63 \frac{N-Z}{A}\right], \\
V_{0}=53 \mathrm{MeV}, R_{0}=r_{0} A^{1 / 3} ; r_{0}=1.27 \mathrm{fm}, a=0.67 \mathrm{fm},
\end{gathered}
$$

and in formula (1) $\kappa$ should be taken in the form

$$
\kappa=0.263\left(1+\frac{N-Z}{A}\right) .
$$

However, calculations of one-particle energies and wave functions with that potential even for spherical nuclei can be carried out only numerically (see, i.e. [11]). A basic drawback of these calculations is that the wave functions are obtained in the form of cumbersome tables; and their use in numerous applications is rather inconvenient (this assertion was absolutely correct 30 years ago, but now, the situation has changed). 
In ref. [12]: Kalinkin, Grabovskii, and Gareev "On levels of mean field of nuclei" (JINR Preprint P-2682; accepted for publication in Acta Physica Polonica on May 23, 1966), we developed an original method of a semianalytic solution to the Schroedinger equation with the realistic potential. According to this method, the radial wave functions can be represented in the analytic form

$$
\psi_{n}(r) \sim \operatorname{const}_{n}(S) \exp \left(-S^{2} / 2\right),
$$

where $n$ are radial wave numbers, $H_{n}$ are Hermite polynomials, and $S(r)$ is a correcting function of the form

$$
S(x)= \begin{cases}b_{1} \ln \left(r / a_{0}\right) & \text { if } r \leq a_{0} \\ b_{2} \ln \left(r / a_{0}\right) & \text { if } r \geq a_{0}\end{cases}
$$

The constants $a_{0}, b_{1}$ and $b_{2}$ are determined numerically with the use of a simple procedure (for details, see [12]).

As it is to be expected, the state wave functions for the realistic potentials differ considerable, and in some cases significantly, from the Nilsson ones, which immediately influences the behavior of many theoretically calculated nuclear characteristics (examples can be found in [13]). Discrepancies are sometimes of an order of magnitude.

Thus, it is completely clear that the prediction of existence of new closed shells in the SHN region can be based only on the study of one-particle spectra constructed on the realistic potential. In this case, to avoid arbitrariness, it is of fundamental importance to base final results on the values of parameters $V_{0}, r_{0}, a$ and $\kappa$ fixed from the analysis of data on independent processes such as excitation of low-lying states of near-magic nuclei, reactions of one-nucleon transfers, processes of elastic and inelastic scattering of nucleons on nuclei, including effects of polarization.

Here it should immediately be stressed that this problem is a particular element in the solution of a more general problem of further verification of the characteristics of the nuclear potential describing averaged "nucleon-nucleus" interactions. The nuclear potential plays a central part in the most of the theoretical models that describe the structure of nuclear states, since it makes the basis one-particle proton and neutron states (energy levels and wave functions) are constructed. Transition to the observable states is accomplished by taking account of the corrections due to the action of residual correlation forces between nucleons (leading, for instance, to the superfluidity effect).

Directions of further verification of the accepted form of the realistic potential are quite clear. One should study its "work" i. in the transition to large values of the mass number A and ii. the possibility of its extension for the transition from the spherical form to the deformed form of a nucleus.

In the first case, this approach corresponds to the study of the SHN region - the prediction and search for new shells, the study of the hypothetical island of stability.

In the second case, this transition is connected with the study of properties of strongly deformed nuclei on the basis of a strongly deformed realistic potential. They include the nuclei of rare-earth and transuranium groups of elements. Note that a positive result in this case is also important for the study of SHN, since it provides a reliable basis for estimations of their fission barriers $V_{f}$ within the framework of shell-correction method (Meyers and Swiatecki [17] formulated first this method and its most consistent form, was developed by V.M Strutinsky [15]). In the next two sections, we consider in more detail the studies performed along these directions. 


\section{The first theoretical predictions of new closed shells with $Z>82, N>126$}

Besides the reasons formulated above, this section is necessary because of the problem of priority. The situation existing in literature confirms this necessity [14].

We present a chronological list of the first theoretical works devoted to the prediction of new shells on the basis of the realistic nuclear potential appeared in the first two years of the corresponding studies. It seems that two years are the most optimal period of time (at least, in those years) for the cycle "study-publication in a journal of general use". Later publications of other authors cannot be considered to be fully independent. For the same purposes, we supply the first works with a more detailed information on the dates when the work was received by a journal and when it was published.

Thus, the first theoretical predictions [2] in accordance with the chronology of their publications with brief commentaries:

1966

1. Gareev F.A., Kalinkin B.N., Sobiczewski A. "Closed Shells for $Z>82$ and $N>126$ in a Diffuse Potential Well", Preprint JINR -2793, Dubna, 1966, published 16 June 1966 and was then distributed by N.I. Pyatov among participants of the Int. Symposium on Why and how should we investigate NUCLIDES FAR OFF THE STABILITY LINE, Lysekyl, Sweden, August 21-27, 1966;

A. Sobiczewski, F.A. Gareev, B.N. Kalinkin, "Closed shells for $Z>82$ and $N>126$ in a diffuse potential well", Phys. Lett. V.22, No 4(1966)500, received 22 July 1966, published 1 September 1966 [2].

In the paper, on the basis of the method developed in our paper [12], the proton and neutron energy levels were calculated in dependence on $A$ at $Z>82$ and $N>126$ for the realistic potential (1-3). The results show that possible magic numbers are $Z=114$ and $N=184$. The calculations were performed with the values of parameters indicated in the previous section. The solution turned out to be stable with respect to the variations of parameters of the potential and spinorbital interaction connected with a possible inaccuracy in their determination. No noticeable energy gap was found in the system of levels near $Z=126$. Also, a rough estimate was given for the fission barrier ( $V_{f} \approx 10 \mathrm{Mev}$ ) of this doubly magic nucleus $Z=114, N=184$. To this end, we used the recipe considered in [16]. The value $V_{f} \approx 7-8 \mathrm{MeV}$ was obtained recently [18] on the basis of a more accurate method of shell correction developed by Strutinsky [19].

This work was the first publication that contains a clear statement on the possibility of a superheavy nucleus with $Z=114$ to exist and expound the method of solution and its stability within the framework of the realistic potential with justified values of parameters.

1967

V.A. Chepurnov" Average field of neutrons and protons shells with $Z>82$ and $N>126$, YaF, 1967, 6, 955 (received 26 February 1967) 20.

In this paper, on the basis of numerical solution to the Schroedinger equation with potential (2) and spin-orbital potential (1) and with the value of parameters practically the same as in [2], systems of proton and neutron levels including cases with $Z>82$ and $N>126$ were obtained. It was confirmed that new shells with $Z=114$ and $N=184$ we have established are realized. Like in [2], no signs of the shell with $Z=126$ to exist were found. This paper contains a reference to [2] and the author mentioned the agreement between his results and ours [2].

In this connection, Chepurnov pointed to a rather instructive result derived in ref. [21]: Wong 
tried to obtain the proton shell with $Z=126$ on the basis of Wood-Saxon potential. He was a success but at what price! He should increase the value of the smearing parameter $a$ by 35 percent, which contradicts its value determined from scattering processes.

Further corroboration of our results was obtained in the following works in 1967:

3. H. Meldner, "Predictions of new magic regions and masses for super-heavy nuclei from calculations with realistic shell model single particle hamiltonians", Proc. of the intern. symposium..., Lysekyl, Sweden, August 21-27, 1966. Received 14 September 1966, published 18 October 1967, Ark. Fys. 36(1967)593 22].

It was reported that new magic numbers should be $Z=114$ and $N=184$, and at the end of the report it was noted that this investigation was carried out independently of our study [2].

4. V.M. Strutinsky, Yu.A. Muzychka, in Proc. of Int. Conf. on Heavy Ion Physics, Dubna, 13-19 October 1966., received on 16 October 1966, published in November 1967, p.51 [23].

5. A.M. Friedman, "Calculations on the production of the next closed shell nucleus and other nuclei" (ibid [24]).

In both the reports, using the realistic potential, the author came to the same conclusion$Z=114$ and $N=184$ are the most pronounced magic numbers in the region of superheavy nuclei.

Thus, the theory based on the realistic potential determined with the help of data on nuclear processes involving spherically symmetric nuclei predicted a nontrivial result: Instead of the earlier expected closed proton shell with $Z=126$ next to $Z=82$, there should be the shell with $Z=114$.

Great significance of this conclusion is quite evident: the experiment on synthesis of the 114th element can be realized considerably easier. Experimenters obtained a well-grounded orienting for organization of the experiment on synthesis of superheavy nuclei [25, 26].

\section{Transition from spherically symmetric nuclei to de- formed ones}

Further development of the conception of the nuclear potential describing the nuclear average field proceeds through its generalization to the case of strongly deformed nuclei.

Success along this line has practical consequences also for solution of the particular problem of search and study of superheavy nuclei:

First, the degree of reliability of the prediction of new shell increases;

Second, the accuracy of estimations rises for the fission barriers of hypothetical SHN;

Third, it makes, in prospect, possible to study the states of deformed SHN.

For the first time, the problem of determination of the one-particle spectrum of deformed axialsymmetric nuclei with the realistic potential was solved by Nemirovskii and Chepurnov [11]. They used the method of numerical integration of a system of differential equations. However, these calculations were complicated and required much computer time (we recall that we are talking about the seventies), and therefore, their further development was stopped.

In this section, we consider another, though approximate, but more effective method of the investigation of one-particle states of deformed nuclei, we have elaborated in [2]. It allowed us to represent the solutions in analytic form, which simplifies their use in applications, and to carry out required calculations more rapidly by several orders. Besides, it was shown in [13] that the energies and wave functions of one-particle states situated near the Fermi surface almost coincide.

Analysis of the rotational bands observed in strongly deformed nuclei and the study of intensities of transitions inside every rotational band and between various bands show that the parity, 
total momentum of a nucleus and its projection onto the intrinsic axis of symmetry are good quantum numbers. And this is possible only in the case when a nucleus possesses axial symmetry. Therefore, it is natural to proceed from the assumption that the dependence of the radius of a deformed axially symmetric nucleus on deformation parameters $\beta_{20}$ and $\beta_{40}$ and angle $\theta$ (counted from the symmetry axis) is of the form

$$
R(\beta, \theta)=R_{0}\left(1+\beta_{0}+\beta_{20} Y_{20}(\theta)+\beta_{40} Y_{40}(\theta),\right.
$$

where $R_{0}$ is the mean radius of a spherical nucleus; $\beta_{0}$ is a constant determined from the condition of nucleus volume being conserved; $\beta_{20}$ and $\beta_{40}$ are parameters of the quadrupole $(\lambda=2)$ and hexadecapole $(\lambda=4)$ deformations. In the main regions of deformed nuclei $150 \leq A \leq 190$ and $230 \leq A \leq 260$ the inequalities $\beta_{20}>0$ and $\beta_{40} \neq 0$ hold valid [27]. Then, for the nuclear potential, we can write

$$
V(\beta, \vec{r})=-\frac{V_{0}}{1+\exp [(r-R(\beta, \theta)) / a]},
$$

(in what follows, for simplicity, we omit indices $N$ and $Z$ of the potential and indices (20) and (40) of the deformation parameters $\beta_{20}$ and $\beta_{40}$. For spin-orbital interaction, the potential should be written in the invariant form

$$
V_{S O}(\beta, \vec{r})=-\kappa[\vec{p}, \vec{\sigma}] \bullet \operatorname{grad} V(\beta, \vec{r}),
$$

where $\vec{\sigma}$ is the spin of a nucleon; and $\vec{p}$, its momentum. Formula (10) transforms into formula (1) when $\beta \rightarrow 0$. For a proton system, it is necessary to add the Coulomb term

$$
V_{C}(\beta, \vec{r})=\frac{3}{4 \pi} \frac{(Z-1) e^{2}}{R_{0}^{3}} \int \frac{n\left(\beta, \overrightarrow{r^{\prime}}\right) d \overrightarrow{r^{\prime}}}{\left|\vec{r}-\overrightarrow{r^{\prime}}\right|}
$$

where the distribution density of the charge in a nucleus $n\left(\beta, \overrightarrow{r^{\prime}}\right)$ equals

$$
n\left(\beta, \overrightarrow{r^{\prime}}\right)=\frac{1}{1+\exp [(r-R(\beta, \theta)) / a]} .
$$

For the spin-orbital interaction (10), we have

$$
\tilde{V}_{S O}(\beta, \vec{r})=V_{S O}(\beta, \vec{r})-V_{S O}(r)=W_{1}+W_{2}+W_{3},
$$

where

$$
\begin{gathered}
W_{1}=-\frac{\kappa}{r} \frac{\partial}{\partial r} \tilde{V}(\beta, \vec{r})\left(p_{\theta} \sigma_{\phi}-\frac{1}{\sin \theta} p_{\phi} \sigma_{\theta}\right) \\
W_{2}=-\frac{\kappa}{r^{2} \sin \theta} \frac{\partial}{\partial \theta} \tilde{V}(\beta, \vec{r}) p_{\phi} \sigma_{r}, \\
W_{3}=-\frac{\kappa}{r} \frac{\partial}{\partial \theta} \tilde{V}(\beta, \vec{r}) p_{r} \sigma_{\phi},
\end{gathered}
$$

where $p_{\theta}, p_{\phi} p_{r}$ are momentum operators in spherical coordinates; $\sigma_{\theta}, \sigma_{\phi}$ and $\sigma_{r}$ are Pauli matrices. It should be remembered that the operator $\tilde{V}_{S O}(\beta, \vec{r})$ is Hermitian, but the operators $W_{1}, W_{2}$ and $W_{3}$ are non-Hermitian, therefore, the neglect of any of them leads to the wave functions being nonorthogonal.

The condition of nucleus volume conservation can be write in the form 


$$
\int n\left(\beta_{00}, \beta_{20}, \beta_{40}, \vec{r}\right) d \vec{r}=4 \pi \int n(\beta=0, r) r^{2} d r .
$$

The equation (15) for determination of $\beta_{0}$ was solved by successive approximation method at the given values of average field parameters.

Denoting the sum of the nuclear and Coulomb potentials and spin-orbital interaction by $V(\beta, \vec{r})$, we perform the identical transformation of the Schroedinger equation

$$
\left[-\frac{\hbar^{2}}{2 m} \Delta+V(\beta, \vec{r})-E\right] \Psi=\left[-\frac{\hbar^{2}}{2 m} \Delta+V(\beta=0, r)+\tilde{V}(\beta, \vec{r})-E\right] \Psi=0,
$$

where

$$
\tilde{V}(\beta, \vec{r})=V(\beta, \vec{r})-V(\beta=0, r) .
$$

Next, let us expand $\tilde{V}(\beta, \vec{r})$ in the series over spherical harmonics

$$
\tilde{V}(\beta, \vec{r})=\sum_{\lambda} C_{\lambda}(\beta, r) Y_{\lambda 0}(\theta)
$$

where the coefficients $C_{\lambda}(\beta, r)$ are determined numerically.

We will look for the solution to equation (11) as the superposition

$$
\Psi_{\nu}(\vec{r})=\sum_{n l j} a_{n l j}^{\nu} \psi_{n l j}^{K}
$$

where $\psi_{n l j}^{K}$ are wave eigenfunctions of the Schroedinger equation with the spherically symmetric potential $V(\beta=0, r)$ :

$$
\left[-\frac{\hbar^{2}}{2 m} \Delta+V(\beta=0, r)-E_{n l j}\right] \psi_{n l j}^{K}=0
$$

with

$$
\psi_{n l j}^{K}=R_{n l j}(r) \sum_{\mu= \pm 1 / 2}(l K-\mu 1 / 2 \mu \mid j K) Y_{l K-\mu} \sigma_{\mu}
$$

where $l$ is an eigenvalue of the orbital moment, $\vec{j}=\vec{l}+1 / 2 \vec{\sigma}, K \equiv j_{z}, n$ is a radial quantum number; and $R_{n l j}(r)$ is a radial part of the total wave function of the form (7). Superposition (19) should contain terms with $l$ of the same parity. Since the potential is axial-symmetric, formula (19) does not contain summation over $K$, and the projection of the angular momentum is in this case an integral of motion.

Substituting expression (19) into (16), multiplying by $\left(\psi_{n^{\prime} l^{\prime} j^{\prime}}^{K}\right)^{*}$ from the left, and integrating, we obtain

$$
\left(E_{n^{\prime} l^{\prime} j^{\prime}}-E\right) a_{n^{\prime} l^{\prime} j^{\prime}}^{\nu}+\sum_{n l j} a_{n l j}^{\nu}<\psi_{n^{\prime} l^{\prime} j^{\prime}}^{K}|\tilde{V}| \psi_{n l j}^{K}>=0 .
$$

Solving the system of equations (22), we can determine the values of energy $E$ and coefficients $a_{n l j}^{\nu}$ of superposition (19), i.e. the wave functions of states.

The expounded approximate method was used to construct the one-particle basis [28] for calculating the characteristics of deformed nuclei in the regions $150 \leq A \leq 190$ and $230 \leq A \leq 260$. Later, in 1970, a complex of programs [29, 30, 31] was worked out for calculations of properties of deformed nuclei in the framework of the Bogoliubov-Soloviev model [32, 33, 34, 35]; and one 
block of that complex calculates one-particle basis states by our method. The complex of programs became a universal tool for concrete computations of the very diverse characteristics of deformed nuclei. Parametrization of the average field and residual interactions turned out to be so successful and adequate to the formulated problem that even 30 years later, this complex is still used at JINR and other nuclear-physics centers, and the characteristics of nuclei calculated on the basis of this model serves as basis for identification and description of experimental data (see, for instance, [36]).

To date, more than a hundred of works is performed and published. The most important results are presented in a series of monographs (see [32, 33, 34]). The general conclusion is that the form accepted in the cycle of our studies for the average field is justified also for the case of strongly deformed nuclei.

\section{$5 \quad$ Realization of the SHN synthesis}

Experimental verification of predictions of the theory could be accomplished only about 30 years later. Synthesis of the ${ }_{114} 289$ element was realized at Dubna at the end of 1998 . It was possible due to purposeful efforts on modification of the accelerating and measuring techniques and the development of experimental procedures. It is quite clear that the very fact of successful synthesis of the 114th element in the reaction ${ }^{48} \mathrm{Ca}+{ }^{244} \mathrm{Pu}$ gives evidence in favor of a great achievement of the experimental nuclear physics. It initiated a chain of new results obtained during the last two years. The following nuclides: ${ }_{114} 287,{ }_{114} 288,{ }_{114} 289$ and ${ }_{116} 292$ were synthesized.

Also, the results of experiments in Berkeley were published [37]. According to these publications, it was possible to observe the chain of decays of the 118th element

$$
{ }^{293} 118 \rightarrow{ }^{289} 116 \rightarrow{ }^{285} 114 \rightarrow{ }^{281} 112 \rightarrow{ }^{277} 110 \rightarrow^{273} \mathrm{Hs} \rightarrow{ }^{269} \mathrm{Sg},
$$

synthesized in the reaction ${ }^{86} \mathrm{Kr}+{ }^{208} \mathrm{~Pb}$.

Of course, it is necessary to carry out further verifications and coordination of these results. However, now we can with high confidence make two conclusions:

1. The prediction of theory [2] based on the approbated realistic potential is experimentally verified: the new proton closed shell with $Z=114$ does exist.

2. The superheavy nucleus 114 (including its isotopes) is not single. In its vicinity, there are other SHN forming the whole region. This result was qualitatively predicted by the theory [39]: if there exists a new closed shell, there should exist the island of stability embracing a certain number of superheavy nuclei around it.

As to the second conclusion, its qualitative character should be emphasized: there is still no theoretically correct procedure of estimation of the SHN lifetime. Therefore, real sizes of the island of stability can be estimated only approximately from general considerations. We formulate them as follows [39]: Concerning the possibility of existence of the island of stability rather than a single superheavy nucleus, in our opinion, evidence comes from the very fact of synthesis of the nucleus with magic number of protons $Z=114$ and nonmagic number of neutrons $N=175$. Indeed, from here, there should follow stability both of the doubly magic nucleus with $Z=114$ and $N=184$ (the island center) and of the nucleus with nonmagic number of protons $Z>114$ but with magic number of neutrons $N=184$. Also, nuclei with values of $\mathrm{Z}$ and $\mathrm{N}$ around the indicated combinations should possess by enhanced stability. Rapid development of studies in this region makes us hope that the validity of this assertion can be verified experimentally in the nearest future. 
To conclude this section, we recall once more that the theoretical prediction of the superheavy nucleus with . to exist, formulated for the first time at Dubna [2] that allowed experimenters to organize its purposeful search, for many years later was confirmed with its actual synthesis at Dubna, too [1].

Unfortunately, this fact is either not reflected in some papers or is distorted.

\section{Conclusion}

What is the resume of the presented discussion? It can briefly be formulated as follows:

1. Conception of the average field used in the modern theory of atomic nucleus and realized in a particular choice of the realistic nuclear potential is further confirmed by the SHN synthesis. The realistic potential works also in the region of extremely large mass numbers $A$. Significance of this fact for further development of theoretical models of the nucleus structure is obvious: if a theory not only explains but also successfully predicts, its reliability as an important element in the construction of nuclear models grows considerably.

It is also evident that the choice of the solution method of the Schroedinger equation with the realistic potential, either semianalytic (as it was done in our works [12]) or completely numerical, is of no theoretical importance, since the results coincide with a good accuracy.

2. Satisfactory description of states of the known strongly deformed nuclei based on the generalized realistic potential makes us hope in its effective use also for studying the states of deformed SHN of the island of stability.

In this connection, it is hoped that the estimate of the fission barrier on the basis of spectra constructed on the realistic potential and of the procedure of shell correction to the drop energy of a nucleus developed by Strutinsky will turn out to be the most consistent and exact.

3. The above consideration shows that the theoretical analysis, which is based on the allowance made for important nuclear physical characteristics, studied before, and which allowed one to predict the existence of long-lived superheavy nuclei in the vicinity of the nucleus with $Z=114$ , and the synthesis of this nucleus and neighboring nuclides, which has recently been carried out , represent a unique study that started 35 years ago and has recently been finished successfully.

The authors are grateful to D.V.Shirkov for attention and support in the development of the problem at JINR.

\section{Appendix}

In section 3, we described real chronology of the first theoretical works devoted to prediction of existence of new closed shells in nuclei with $Z>82$ and $N>126$. As we indicated in the Introduction, some authors of recent publications admit considerable inaccuracies, errors, and even distortions of the known historical facts. Below we present only two examples.

G. Herrmann in the review paper "From Nuclear Fission to Superheavy Elements" (匹4, p.14) wrote:

...Heiner Meldner (Arkiv Fysik 36(1967)593) showed that shell closedes should be expected at proton number 114 and neutron number 184, not too far from the then heaviest elements and, thus, perhaps within rich...

It is difficult to imagine that the author of this review does not know about our paper [2] — it is published in the well-known journal — "Physics Letters". Even more, there is reference to our 
paper in H. Meldner's paper quoted by the author. There are references to our publication even in textbooks [32, 38] (see details in [39]).

Another example is the review by S.Hofmann and G.Munzenberg "Discovery of the heaviest elements" (Rev. Mod. Phys., 72, 3, July, 2000). We quote only one sentence, in which the authors discuss principal important questions (page 734):

The prediction of magic numbers was less problematic than the calculation of the stability of those doubly closed shell nuclei against fission...

A series of papers have based calculations of the location and properties of superheavy elements (SHE's) on the Strutinsky shell-correction method (Myers and Swiatecki, 1966; Meldner, 1967; Nilsson et al., 1968; Mosel and Greiner, 1969; Fiset and Nix, 1972; Randrup et al., 1976)...

The first affirmation rouses astonishment. The reliability of predictions of shell effects in the spectrum of one-particle nuclear states and the origin of the energy gap depend on the substantiation of the choice of the average nuclear field and on its values of parameters to be fixed established by the analysis of independent nuclear reactions (a wide range of direct nuclear reactions, elastic and inelastic scattering of particles on nuclei, the data from nuclear spectroscopy). It is well-known that localization of new shells essentially depends on the form and parameters of a nuclear potential. As for the stability of heavy nuclei, both those existing already and those to be predicted, it mainly depends on the behaviour of "a shell correction" part to "a drop" part of nuclear energy depending on its deformation (according to Meyers and Swiatecki; Strutinsky). The calculation of "the shell correction" is by a concrete shell scheme, and so, in spite of its importance, it is secondary (it is clear from the term - "shell correction").

As it was shown in a preprint by B.N. Kalinkin and F.A. Gareev [39], the first prediction for a new proton shell with $\mathrm{Z}=114$ arising in state spectra for a realistic potential with well-founded values of parameters and spin-orbital interaction was formulated in papers by F.A. Gareev, B.N. Kalinkin, A.Sobiczewski, Preprint JINR P-2793, June, 1966; A.Sobiczewski, Gareev F.A., B.N. Kalinkin, Phys. Lett., 22, No.4, 1966, p.500. The estimation of fission barrier $V_{f}$ for a double magic nucleus with $\mathrm{z}=114$ and $\mathrm{N}=184$ was also given in this work $\left(V_{f}=10 \mathrm{MeV}\right)$. Unfortunately, the authors of the review give reference to this paper only for the discussion of a particular case - the question about spin-orbital interaction in nuclei removing it in background.

So, the second confirmation including reference to be contained in it completely twists the realistic situation and well-known facts.

First, Meyers and Swiatecki don't predict new magic numbers. For demonstration, we quote fragment of their paper:

We do not wish to imply that there are grounds for believing that any of these magic numbers $(Z=126, N=184,258)$ would show up in practice, and we use them only to illustrate that some of the consequences would be if a magic number turned out to be present in the general neighborhood of super- heavy nuclei somewhat beyond the end of the periodic table. The actual values of the magic numbers might be different...

...In order to proceed in a realistic manner with discussion of the existence and location of a possible island of stability beyond the periodic table, the first requirement is the availability of estimates for the location and strength of magic number effects in that region. When such estimates have become available (through single - particle calculations in realistic nuclear potentials), it will be possible to apply our semi-empirical treatment of nuclear masses and deformations to the predictions of the fission barriers of hypothetical super - heavy nuclei.

We see, Myers and Swiatecki in their calculations used the values of magic numbers obtained by other authors with the harmonic potential. Estimates on the basis of realistic potentials were not available for them that time. 
Second, Myers and Swiatecki don't use the Strutinsky-method as the review authors state. Strutinsky published his method in 1967, whereas Meyers and Swiatecki developed their method earlier(UCRL 1965 Nuclear Phis.,1966). Moreover, the method (Meyers and Swiatecki) was severely criticized by Strutinsky ([40], p. 526, see also [19], p. 420):

Recently Swiatecki has done an interesting attempt of phenomenological description of shell effects and deformation in the nuclear mass (W.D. Myers, W.J. Swiatechi. Nucl. Masses and Deformations, UCRL-11980 (1965), W. Swiatechi. Proc. 2d Int. Conf. on Nuclidic Masses, Spr.Verlag, Viena, p. 58(1964)). Based on simplification of the level model in deformed nuclei and assumption of shell disappearance at some value of the deformation parameter $\alpha_{0}$, an expression was proposed of the type $S(N, Z) \sim \exp \left[-\left(\alpha / \alpha_{0}\right)^{2}\right]$ by him for describing the correction to the "drop" mass of a nucleus. This method would be very comfortable but, unfortunately, a more complex expression is required for the shell effect description. So ,it is easy to see that, in deformed nuclei in the Swiatecki model, we always have a positive mass correction and the threshold of nuclei fission less than the drop one and more strongly dependent on $Z^{2} / A$ than in the ordinary drop model. Following our calculations, the shell correction $\delta V$ in the middle of the shell for deformed nuclei is negative and is large in magnitude up to $\approx 3 \mathrm{Mev}$. So, because of the restriction of the expression for correction in the Swiatecki model, practically, we have the same energy for flattened and stretched states. And this is obviously wrong.

The third moment is that Meldner, despite the authors' desire, doesn't consider the dependence of the one-particle spectrum on deformation, and hence, in his paper, he did not use the Strutinsky method and, moreover, did not estimate the fission barrier for superheavy nuclei. So, he did not investigate the problem of their stability.

In accordance with arguments given above, we assume that there is the following true formulation for the second confirmation: In a series of papers, calculations were performed for the location and properties of newly predicted super heavy elements (Sobiczewski, Gareev and Kalinkin, 1966; Chepurnov, 1967; Meldner, 1967; Nilsson et al., 1968; Mosel and Greiner, 1969; Fiset and Nix, 1972; Randrup et al., 1976.

We emphasize once more that our paper was the first prediction of the existence of nuclei in a new shell $\mathrm{Z}=114$ and $\mathrm{N}=184$ based on the realistic potential and numerous data on nuclear reactions. The first estimation of the barrier fission for this nucleus was also done. Its value is close to recent calculations [18]. A detailed evidence of priority of this paper was given in [39].

It is a pity that the authors of the review devoted the whole chapter to the problem of denomination of new elements and did not pay any attention to principal questions. 


\section{References}

[1] Oganessian Yu. Ts. et al., Preprint JINR E-7-99-53, Dubna, 1999; YaF, 2000, 63, 1769; Phys. Rev., 2000, C62.0411604(R); Phys. Rev. Lett., 1999, 83, 3154; Nature (London) 1999, 400, 242; Europhys. Jour. 1999, A5, 63.

[2] Gareev F.A., Kalinkin B.N., Sobiczewski A.//Preprint JINR P-2793, Dybna,1966; Phys. Lett. 1966, 22, 500 .

[3] Oganessian Yu. Ts.//JaF, 2000, 63, 1391; Nucl. Phys., 2001, A682, 108.

[4] Herrmann G.//Nuclear Physics News 1998, 8, No.2, 7.

[5] Hofmann S., Munzenberg G.//Reviews of Modern Physics, 2000, 72, 733.

[6] Nemirovski P.E.//Sovremennue modeli atomnogo jadra. M.: Atomisdat, 1960.

[7] Preston M.A.//Physics of Nucleons, Addison-Weslag Pub. C., Jnc., Reading, Massachusetts, Palo-Alto-London, 1962.

[8] Nilsson S.G.//Kgl Danske Vidensk, Selsk. mat-fys. Medd. 22, N 16, 1955; Mottelson B.R., Nilsson S.G.//Kgl Danske Vidensk, Selsk. mat-fys. kr. 1, N 8, 1959.

[9] Heisenberg V.//Teoriya atomnogo yadra, IL, Moscow, 1953.

[10] D. Saxon, R. Wood, Phys. Rev. 95, 577, 1954.

[11] Nemirovski P.E., Chepurnov V.A.//YaF 1966, 3, 998.

[12] Kalinkin B.N., Grabovski Ja., Gareev F.A.// Preprint JINR P-2682, Dubna, 1966; Acta Physica Polonica, 1966, XXX, No.6, 999.

[13] Grabovski Ja., Kalinkin B.N.// Nucleonica, 1967, XIV, 571.

[14] Kalinkin B.N., Gareev F.A.//Nuclear Physics News, 2001, 11, No.2; LANL nucl-ex/0105021.

[15] Strutinsky V.M., Received 14 September 1966, published 18 October 1967, Ark. Fys., 1967, 36, 629; in Proc. of USSR Summer Scool "Nuclear Spectroscopy by Nuclear Reactions", Obninsk, 3-12 July, 1966.

[16] Swiatecki W.J., in Proc. Second Int. Conf. on Nuclidic Masses, Vienna, 1963, Springer-Verlag, Vienna, 1964, p.58;Meyers W.D. and Swiatecki W.J., Report UCRL-11980, 1965.

[17] Meyers W.D., Swiatecki W.J.//Nucl. Phys., 1966, 81, 1.

[18] Smolanczuk R., Sobiczewski A.,// in Proc. of the XV Nuclear Physics Divisional Conference in Low Energy Nuclear Dynamics, St. Petersberg, Russia, April 18-22, 1995, edited by Yu. Oganessian, R. Kalpapakchieva and von Oertzen (World Scientific, Singapore), p. 313.

[19] Strutinsky V.M.,//Nucl. Phys., 1967, A95, 420.

[20] Chepurnov V.A.,//YaF, 1967, 6, 955.

[21] Wong C.Y.//Phys. Lett., 1966, 21, 688. 
[22] Meldner H.,//in Proc. of the intern. symposium..., Lysekyl, Sweden, August 21-27, 1966. Received 14 September 1966, published 18 October 1967, Ark. Fys. 36, 593, 1967.

[23] Strutinsky V.M., Muzyka Yu.., //in Proc. of International Conf. in Heavy Ion Physics, Dubna, 13-19 October 1966, received on 16 October 1966, published in November 1967, p.51.

[24] A.M. Friedman, //in Proc. of International Conf. in Heavy Ion Physics, Dubna, 13-19 October 1966, received on 16 October 1966, published in November 1967, p.39.

[25] Kalinkin B.N.// Preprint JINR E4-3792, Dubna, 1968.

[26] Grabovski Ja., Kalinkin B.N.//Nucleonica, 1969, XIV, No.6, 584.

[27] Gareev F.A., Ivanova S.P., Paschkevich V.V.//YaF, 1970, 11, 1200.

[28] Gareev F.A., Ivanova S.P., Kalinkin B.N.//Acta Physica Polonica, 1967, 32, 461; 1968, 33, 133; Izv. AN SSSR, ser. fiz., 1968, bf 32, 1690.

[29] Gareev F.A, Ivanova S.P., Malov L.A., Soloviev V.G. //Nucl. Phys., 1971, A171, 3.

[30] Gareev F.A., Ivanova S.P., Soloviev V.G., Fedotov S.I.// Particles and Nuclei, 1973, 4, 355.

[31] Gareev F.A., Ivanova S.P., Schirikova N.Yu.//Communication JINR 4-5457, Dubna, 1970; Preprint JINR E4-4259, Dubna, 1969.

[32] Soloviev V.G.//Teorija slozhnux jader. M.: Nauka, 1971.

[33] Grigorev E.P., Soloviev V.G.//Struktura chetnux deformirovannux jader. M.: Nauka, 1974.

[34] Soloviev V.G.//Teorija atomnogo jadra. Jadernue modeli. M.: Energoizdat, 1989.

[35] Soloviev V.G.//Teorija atomnogo jadra. M.: Energoizdat, 1989.

[36] Ahmad I et al.,//Phys. Rev., 2000, C62.064302, 1.

[37] Ninov V., et al., //Phys. rev. Lett., 1999, 83, 1104.

[38] Frauenfelder H. Henley E. Subatomic Physics. Prentice-Hall, Englewood Cliffs, New Jersey, 1974.

[39] Kalinkin B.N., Gareev F.A.//Preprint JINR E7-99-107, Dubna, 1999.

[40] Strutinsky V.M., Proc. of USSR Summer Scool "Nuclear Spectroscopy by Nuclear Reactions", Obninsk, 3-12 July, 1966. 